\title{
Dense Fractal Networks, Trends, Noises and Switches in Homeostasis Regulation of Shannon Entropy for Chromosomes’ Activity in Living Cells for Medical Diagnostics
}

\author{
Nikolay E. Galich \\ Department of Experimental Physics, Saint-Petersburg State Polytechnic University, Saint-Petersburg, Russia \\ Email: n.galich@mail.ru
}

Received July 7, 2013; revised August 7, 2013; accepted August 15, 2013

Copyright (C) 2013 Nikolay E. Galich. This is an open access article distributed under the Creative Commons Attribution License, which permits unrestricted use, distribution, and reproduction in any medium, provided the original work is properly cited.

\begin{abstract}
We analyze correlations and patterns of oxidative activity of 3D DNA at DNA fluorescence in complete sets of chromosomes in neutrophils of peripheral blood. Fluorescence of DNA is registered by method of flow cytometry with nanometer spatial resolution. Experimental data present fluorescence of many ten thousands of cells, from different parts of body in each population, in various blood samples. Data is presented in histograms as frequency distributions of flashes in the dependence on their intensity. Normalized frequency distribution of information in these histograms is used as probabilistic measure for definition of Shannon entropy. Data analysis shows that for this measure of Shannon entropy common sum of entropy, i.e. total entropy $E$, for any histogram is invariant and has identical trends of changes all values of $E(r)=\ln r$ at reduction of rank $r$ of histogram. This invariance reflects informational homeostasis of chromosomes activity inside cells in multi-scale networks of entropy, for varied ranks $r$. Shannon entropy in multi-scale DNA networks has much more dense packing of correlations than in "small world" networks. As the rule, networks of entropy differ by the mix of normal $D<2$ and abnormal $D>2$ fractal dimensions for varied ranks $r$, the new types of fractal patterns and hinges for various topology (fractal dimension) at different states of health. We show that all distributions of information entropy are divided on three classes, which associated in diagnostics with a good health or dominants of autoimmune or inflammatory diseases. This classification based on switching of stability at transcritical bifurcation in homeostasis regulation. We defined many ways for homeostasis regulation, coincidences and switching patterns in branching sequences, the averages of Hölder for deviations of entropy from homeostasis at different states of health, with various saturation levels the noises of entropy at activity of all chromosomes in support regulation of homeostasis.
\end{abstract}

Keywords: Abnormal Fractals; DNA Activity and Shannon Information Entropy; Fractal Patterns and Fragmentation; Informational Homeostasis; Saturations of Chromosomal Correlations; Multi-Scale Fractal Networks of Shannon Entropy

\section{Introduction}

We oriented on medical diagnostics of health status based on oxidative activity of DNA in cells for everyday clinical practice, for given person at given time. Oxidative activity of DNA is visualized in fluorescence. We analyze experimental data on DNA fluorescence in neutrophils of peripheral blood at biochemical reaction of oxidative burst [1]. This is a high sensitive method for diagnosing many different and complex diseases, early diagnostics of illnesses, hidden diseases. Short list of clinical observations is given in [1-4]. Preparation and experimental procedures, including small additions of ethidium bromide for small volumes of blood $\sim 2 \mathrm{ml}$, excitation and measurements of fluorescence are described in [1-6]. DNA fluorescence is described by histograms in flow cytometry method with spatial resolution of measurements at a few nanometers in the flow direction $[5,6]$. Statistics of fluorescence is presented in histograms for frequency of flashes $P(I)$ as the functions of fluorescence intensity I for large populations of many ten thousands of 
cells, living in different parts of human body. Chaotic Brownian motion and rotation of fluorescing cells, flowing through the laser beam in flow cytometry, ensured good statistics for illustration of real 3D chromosomal activity inside living cells. Accuracy and reproducibility of experimental results in histograms of fluorescence approximately equal to $2 \%$ that corresponds to the normal, usual levels of inevitable and fatal errors and fluctuations of physical and biological nature [1-6]. Three original histograms, as the illustrations of typical examples, are shown in Figure 1.

The heterogeneous fluorescence of all chromosomes in the cells reflects simultaneously the genetic special, individual features and immune response to the pathogenic actions due to oxidative activity of DNA. Detailed accurate statistical analysis of these histograms currently is absent. Large-scale correlations for distributions of fluorescence flashes of DNA inside living cells differ from those that we would like to see by abnormal fractal dimensions and non-trivial noise level at substantially nonGaussian statistics [3-6]. These natural peculiarities of immunofluorescence are often accompanied by statistical instabilities of local intensity distributions [3-6], i.e. fast exponential growth of central moments of fluorescence intensity. We need to develop a sequence of new nonlinear statistical methods for data analysis of immunofluorescence. Standard smoothing eliminates destroys and removes various peculiarities of fractal networks and correlations in the activity of DNA, changes real statistics, blurs and distorts many aspects of reality.

According to tradition, now and in the latter time, dominating sciences about DNA based on various approaches in biochemistry, structural biology, materials science and combinatorics for lonely DNA. In this case,

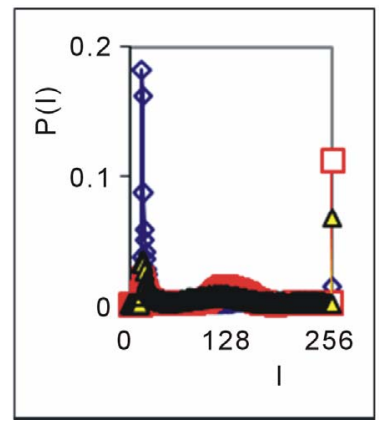

(a)

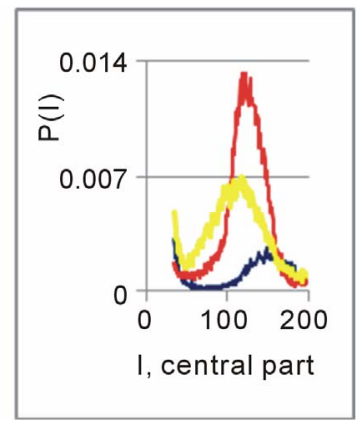

(b)
Figure 1. Dependence of normalized frequency distribution of flashes $P(I)$ on their intensity $I(a)$; for clearest, only central part of histogram (b). The area under the final histograms of $P(I)$ normalized to unit; rhombus points correspond to bronchial asthma. Total number of flashes is $N_{0}=$ 76 623; quadrate points correspond to the healthy donor. Common number of flashes is $N_{0}=40109$; triangle points correspond to the oncology disease. Common number of flashes is $N_{0}=40752$. informational activities of DNA in real life inside living cell, for full set of 3D chromosomes, their overall communications and information flows, switches in topology and informational networks in the presence of real changeability and noises in information transfer are lost, go off, pushed back far into the background, as the basic unsolved problems. What it means for information transfer of DNA activity inside living cells, for diagnostics and comparative analysis of health and diseases?

In Part 2 of this article we defined informational homeostasis of Shannon entropy as the empirical result. In Part 3 we analyze a density of packing, new types and classes of fractal networks of DNA entropy. In Part 4 we analyze deviations of entropy from homeostasis and various switches for central moments and averages of Holder at variations regulation of homeostasis for different states of health. We observe saturation for averages of Holder if the number of averages coincides with full chromosome number; all chromosomes are involved in support of regulation of informational homeostasis. The levels of saturations have various switching at changing the state of health. In Part 5 we show that this switching connected with stability change in homeostasis regulation. We show that all distributions of information entropy are divided on three classes, which associated in diagnostics with a good health or dominants of autoimmune or inflammatory diseases. This classification based on switching of stability at transcritical bifurcation in the nonlinear dynamical system of homeostasis regulation.

\section{Information on Oxidative Activity of DNA inside Cells. Shannon Entropy. Informational Homeostasis}

Oxidizing activity of DNA in the cells activates various (all possible) correlations with different combinations of non-coding and coding DNA fragments, both within one and the same chromosome, and between different chromosomes. Actual, 3D topology of chromosomal correlations in the nuclei of cells has quite notable changes over time, during only one month, for cells living inside given human, in the process of human life or at diseases development, during medical treatment, etc. [1-6]. Characteristics, changes and deviations various fractal correlations in networks of fluorescing DNA inside cells can be used for medical diagnostics [6]. How these results may to use for definition varied structures of informational activity of DNA?

We all, all our chromosomes and all our cells are the open systems. How to estimate quality, quantity and changeability of DNA communications and DNA information transfer in life of given person? What about information $J=-\ln P$ and information entropy for DNA activity inside cells? What need for comparison various data on informational activity of DNA inside cells for 
one and the same human at various times and for different people? Here we have no clear criteria. How to determine the existence and level of information noise, switches, changeability and stability of information transfer inside living cells for any being? What it means for medical diagnostics from the point of view different types of oxidative metabolism of 3D DNA inside cells, for inner and inter chromosomal correlations at different states of health? The answers on these questions now are absent, that associated with deep and unsolved problems in fundamental mathematics, information theory, etc.

To diagnostics features oxidizing activity of DNA in the blood cells for one and the same person at different times or in blood cells of different people need to compare various distributions of fluorescence, which are very diverse and changeable, as for three examples in Figure 1. A huge role here is played by the irregularity, infrequency and brokenness of histograms, which define basic information on DNA activity [5-7]. Any artificial smoothing results destroy this information. For comparison various distributions of fluorescence we must understand the origin and function of these distributions under what conditions and parameters they need to be compared. For example, at Gaussian distribution of random variables is important to know only the mean and variance. If the statistics is very complex, such parameters and their combinations can be very, very, many. Here observed non-trivial noises of fluorescence and the exponential divergence central moments for fluctuations of intensity at increasing the number of central moments [3-5]. This is a clear sign of turbulence [3]. In this case, when comparing different distributions and moments for fluctuations of intensity the number of corresponding moments also exponentially quickly grows with increasing the order of diverging statistical moments. The similar procedures of comparisons haven't the sense in nature and in science, excluding examples and illustrations the growth for rate of statistical instabilities in the interpretation of complexity. Reproducible results and clear analysis real activity various DNA inside cells produce the need of clear, stable levels and criteria for comparison different distributions of DNA fluorescence.

Let us introduce frequency distribution of Shannon entropy $E_{l}=-p_{i} \ln p_{i}$, based on the frequency distributions of information $J_{l}=-\operatorname{Ln} P_{l}$ (see Equations (1)-(3)). Three examples of frequency distributions of Shannon entropy $E_{l}=-p_{i} \ln p_{i}$, based on the frequency distributions of information $J_{l}=-\operatorname{Ln} P_{l}$ in Figure 2(a), are shown in Figure 2(b). We present comparison of distributions of immunofluorescence based on the universal, empirical invariant of information entropy $E(J, r)=$ $\ln r$ at given rank $r$. Rank $r$ is defined by the maximal number of measuring channels $I_{\max }=r$. Detailed definition of empirical invariant of information entropy
$E(J, r)=\ln r$ is presented below, in Equations (4)-(7). This empirical invariant shown in Figure 2(c), as only one, overall numerical value of total Shannon entropy $E(J, r)=\ln r$ for given rank $r$, for DNA fluorescence inside any neutrophils, living in any people with different states of health.

This invariant was observed during fluorescence of DNA in human neutrophils in different samples of blood [5,7], as in Figure 2(c). We observe only one unified value of total Shannon entropy $E(J, r)=\ln r$, like the empirical invariant, as the identical sum in each given distribution of entropy, in given sample of blood, for all cells and any donor. This invariant has one and the same value of total Shannon entropy $E(J, r)=\ln r$ at fluorescence for given rank $r$ of histograms, i.e. given scale

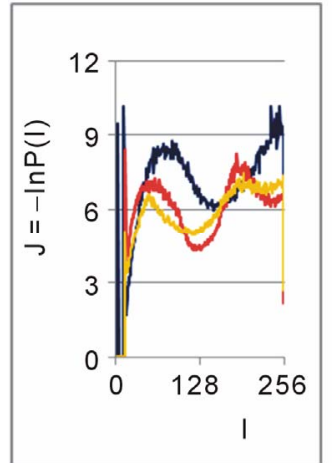

(a)

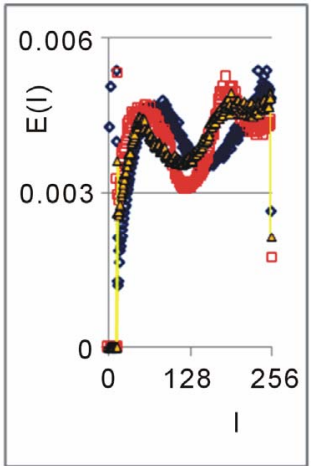

(b)

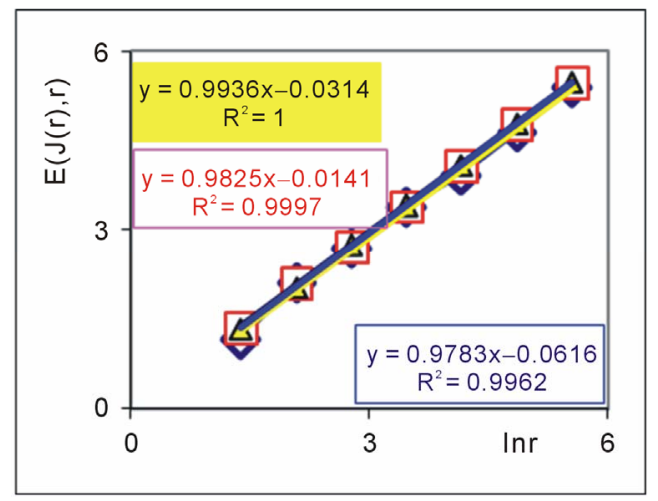

(c)

Figure 2. (a) Logarithmic dependence $\operatorname{Ln} P(I)$ for frequency of flashes $P(I)$ on their intensity $I(r=256)$; The area under the initial histograms of $P(I)$ normalized to unit; Original histograms for $P(I)$ shown in Figure 1; (b) Normalized distributions of information entropy $E(J(I))$ in the dependence on fluorescence intensity $I(r=256)$; rhombuses correspond to bronchial asthma. Total number of flashes is $N_{0}=76$ 623; squares correspond to the healthy donor, $N_{0}=$ 40 109; cross correspond to the oncology disease. Common number of flashes is $N_{0}=40$ 752; (c) Dependence of total Shannon information entropy $E(J, r)=\ln r$ on logarithm of range $r$; initial histograms at range $r=256$ shown in Figures 1(a) and 2(b). 
of clusters in networks of DNA entropy. This invariant defines the informational homeostasis of oxidative activity of 3D DNA for full set of chromosomes inside living cells, at any biodiversity of cells for Shannon-Weaver index [5]. This invariant gives the overall zero level for countdown of information activity of DNA in cells for any person, at any state of health and for any genome of different people.

In Figure 2(a) we see a very strong roughness and differences in information $J(I)=-\operatorname{Ln} P(I)$ over a wide range of changing the order of values $J(I)$ from zero up to ten; typical level of information is about $J \sim 7$. High level of noises instead of a smooth continuity for all local distributions of information $J(I)=-\operatorname{Ln} P(I)$ reflects main natural properties of DNA activity, which provide main correlations in oxidative activity of DNA inside cells, in gene's networks, in metabolism and cell viability. Any forced smoothing of experimental data here hampering any our attempts to adequate perception of real life DNA inside living cells and violates the homeostasis of entropy.

Let us consider the probability density $P(I)$. i.e. frequency distribution $P_{l}$ the number of flashes, $P_{l}=N(l) / N_{0}$, where $l$ is the number of measuring channel, $l=1,2, \cdots, 256 ; \quad N_{0}$ is the total number of flashes; $N=N(l)$ is the number of flashes with the assigned intensity $I=l$; dimensionless intensity $I$ coincides with the number of measuring channel l, i.e. $I=l$; $\langle P\rangle=\left(I_{\max }-I_{\min }\right)^{-1}$ is the mean probability value; symbol $\langle\ldots\rangle$ denotes the statistical average for number flashes of fluorescence in all of $r=256$ channels of intensity measurement. The mean value of $\langle P\rangle$ is equal to $1 / 256$ for $r=256$ channels of intensity measurement. Three examples of frequency distributions of $P_{l}$ for different donors with varied states of health are shown in Figure 1.

Distribution of information $J_{l}$ defined as

$$
J_{l}=-\operatorname{Ln} P_{l}
$$

Let us consider the normalized distribution of information

$$
p_{l}=J_{l} / \sum_{l=1}^{l=256} J_{l}
$$

as the probabilistic measure for frequency distributions of Shannon entropy $E_{l}$

$$
E_{l}=-p_{l} \ln p_{l}, E(J(I))=E\left(J_{l}\right)=E_{l}
$$

Data analysis of all experiments has shown the conservation of total Shannon entropy

$$
E(J)=\sum_{i=1}^{i=256} E_{i}=\text { const }
$$

Thus, total Shannon entropy $E(J)$ is empirical invariant, for all neutrophils in all donors [5,7]. Value of total entropy $E(J)=E(J, r)=\operatorname{const}(r)$ depends on given rank $r$ of histogram (see Figure 2(d) and Equation (7)). Rank $r$ is defined by the maximal number of measuring channels $I_{\max }=r$. At rank $r=256$ all experimental data, for all donors give one and the same value of $E(J, r=256)=5.48$ with standard deviation $\sim 2 \%$, within limits of typical for flow cytometry experimental errors $\sim 2 \%$ at 256 measuring channels $[1,2]$. Decreasing maximal number of channels or rank $r$ leads to decreasing the value of invariant $E(J, r)$. Three illustrations of informational homeostasis for distributions of information $J_{i}=-\operatorname{Ln} P_{l}$ and entropy in Figures 2(a) and (b) are shown in Figure 2(c) at different rank r. Other examples of informational homeostasis for different patients with various diseases had shown in [5].

Invariance of total entropy $E(J, r)$ defines special role of distribution of Shannon entropy $E(J(I))$, as is for all functions, associated with the conservation laws, as the main dominant variable to describe the states and dynamics of informational activity of DNA inside cells.

How will be changing the information $J(I)$ due to reduction of rank $r$ or the range $r$ at definition of Shannon entropy? Here, as everywhere, are used different terms a rank $r$ and range $r$ for the same value of $r$. Range of histogram $r$ interconnected with the selection of multistage clusters in networks with structure of bronchial tree; here range $r$ coincides with the number of columns in a histogram or with the number of channels for measurements of fluorescence intensity at given maximal value of dimensionless intensity, i.e. $r=I_{\max }$. In our experiments the number of channels is $r=256$. Variations of range $r$, i.e. rank of histogram $r$, or variations the scale $r$, when $r=I_{\max }$, provide the changes in irregularity and brokenness of frequency distribution of fluorescence for histograms of various rank $r$. Various examples decreasing of histograms rank $r$ presented in $[3,5,7]$. Integer $r$ defines the total range $r$ for distribution of entropy as maximal number of columns in reduced histogram. Each reduction of $r$ leads to the redistribution of probability density $P(I, r)$ and information $J(I, r)$. Reduced distribution of probability $P(I, r)$ described in [3]. Reduced distribution of information is $J(I, r)=-\ln P(I, r)$. Normalized frequency of information $p_{l}(r)$ during reduction of range $r$ is

$$
p_{l}(r)=J_{l}(r) / \sum_{l=1}^{l=r} J_{l}(r), J_{l}(r)=-\operatorname{Ln} P_{l}(r)
$$

Frequency distribution of entropy $E_{l}(r)$ for an arbitrary rank $r$ is

$$
E_{l}(J, r)=-p_{i}(r) \ln p_{l}(r)
$$

Total entropy $E(J, r)=-\sum_{l=1}^{l=r} p_{l} \ln p_{l} \quad$ is invariant 
identical for given $r$ in all cells. Total entropy $E(J, r)$ depends only on rank $r$, as it is shown in Figure 2(c). Dependence of $E(J, r)$ on rank $r$ in Figure 2(c) is logarithmic

$$
E(J, r)=-\left\langle\ln p_{i}(r)\right\rangle--\sum_{l=1}^{l=r} p_{l} \ln p_{l}=\ln r
$$

Informational homeostasis of total Shannon entropy $E(J, r)=\ln r$ for oxidative activity of DNA is observed in all cells of blood different old and young patients with various states of health and all the time. It means existence of informational homeostasis of DNA during all the life time of cells from birth to death.

\section{Manifold of Dense Fractal Networks of Shannon Entropy for Information}

Let us consider some fractal peculiarities of immunofluorescence distribution. Different analogies of fractal networks such as bronchial tree, structure of oncology tumor, arterials tree, etc with networks and distributions of immunofluorescence are described in [3]. Many histograms of different origin are the similar to the histograms for fluorescence of neutrophils in Figure 1 [3]. Range of histogram $r$ interconnected with the selection of multistage clusters in networks with structure of bronchial tree. Variations of range $r$, i.e. rank of histogram $r$, or variations the scale $r$ provide the changes in irregularity and brokenness of frequency distribution of fluorescence for histograms of various rank $r$. The quantitative measure of irregularity and brokenness for frequency distribution of flashes for any rank $r$, in all histograms may serve a Hurst index Н. может служить.

Hurst exponent $H$ [8] is determined by means of regression equation

$$
\operatorname{Ln}(R / S)=H \times \operatorname{Ln} I+\text { const }
$$

where $R / S$ is rescaled range $(R=S), R$ is range or maximal deviation of $P(I)$ from local mean level, $S$ is standard deviation of $P(I)$. Illustration of definition Hurst index was presented in $[5,6]$. Hurst index $H$ for frequency of flashes $P(I)$ corresponds to fractal (Hausdorff) dimension $D[8]$ if

$$
D=2-H
$$

In Figure 3 presented three distributions of fractal dimensions $D$ for frequency distributions of Shannon entropy in Figure 2(b).

As the rule, networks of entropy are characterized by a mix of normal $D<2$ and abnormal $D>2$ fractal dimensions, as in Figure 3. Abnormal fractals $D>2$ are typical for entropy' networks in a good health and for oncology at different values of rank $r$ and perfectly absent at bronchial asthma, where $D<2$ for all rank $r$. Networks of

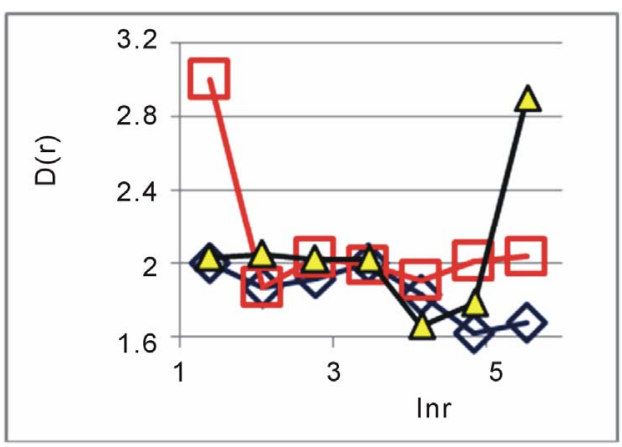

Figure 3. Dependence of fractal dimension $D(r)$ on logarithm of range $r$ in networks of Shannon entropy with different scales for three different states of health connected with asthma, with good health and at oncology; initial histograms shown in Figure 2(b).

information entropy for DNA fluorescence are formed by normal and abnormal fractals. Normal fractal dimension $D$ corresponds to interval $1<D<2$ and positive Hurst index $H>0$. Negative Hurst index $H<0$ gives the anomalous fractal dimensions $D=(2-H)>2$. Absolute majority of the authors ignore any anomaly fractal dimensions. Nonetheless, negative Hurst index $H<0$ does not contradict of main definitions of the power-law correlations for fractal distributions [9], subject to rejection from the hypothesis of self-affinity. In the case of $H$ $<0$ an anomalous fractal dimension $D>2$ can serve as a measure of fragmentation for correlations in complex networks [10].

Abnormal fractal dimensions $D>2$ give not only fragmentation of correlations but also ensure more dense packing of fractal networks. The size of all and any fractal clusters $d \sim N^{1 / D}$, for certain number of nodes $N$, decreases at increasing the value of $D$. We do not know characteristics of fragmentation of information entropy of DNA and we do not have the recipes and methods of its descriptions. Therefore, we guide by clear signs of new peculiarities and strong expressions of contradictions with the traditional images of modern standards, diagrams and descriptions of DNA activity inside cell. For example, we consider different unusual deviations of typical features from very popular networks of "small worlds" [11], that are often used to describe global DNA activity inside cells $[12,13]$.

According to Figure 3 there is no unambiguous and simple connection of network topology or fractal dimension $D$ with the certain value of information entropy $E(J, r)=\ln r$ at informational homeostasis.

Consider an undirected network, and let us define $\mathrm{d}$ as the mean geodesic (i.e., shortest) distance between pairs of vertex or nodes in a network of flashes of fluorescence. The certain number $N$ of synchronized nodes-flashes in networks of DNA fluorescence inside cells are characterized by the intensity $I \sim N$, where $N$ defines a common 
number of correlated nodes in network, if every node in fluorescence network has the approximately identical fragment of oxidative activity of DNA with approximately identical quantity of fluorescing dye. More detailed determination of correlated nodes $\mathrm{N}$ in the clusters of fluorescence networks of DNA inside cells now is unknown. The correlation length $d$ depends on the network topology. Random networks with a given degree distribution may be the networks of "small worlds" [14], as in one from most popular family of complex networks [11-13]. "Small world" behavior is typically characterized by logarithmic scaling for path length tends $d \sim \ln N$ [14]. On the other hand the expression of $d \sim N^{1 / D}$ defines a linear size of D-dimensional lattice or the size of a fractal cluster $d \sim N^{1 / D}$. Therefore estimation of fractal dimension $D$ of fluorescence in the networks of "small worlds" is $D(N) \sim \ln N / \ln \ln N$. Standard definition of fractal dimension $D[8,10]$

$$
D=\lim _{d \rightarrow 0}(\operatorname{Ln}(N(d)) / \operatorname{Ln}(d))
$$

also gives $D(N) \sim \ln N / \ln \ln N$ in "small worlds" network. We use various experimental data in histograms to define Hurst index $\mathrm{H}$ and fractal dimension $D$ according to Equations (8)-(10). The transformation of "small worlds" due to reduction of range $r=I \sim N$ leads to expression $\ln r \sim D \ln \ln r$. In these variables data in Figure 3 are transformed to Figure 4(a). Using of variables for networks of "small worlds" gives varied distributions of fractal topology for Shannon entropy which have a view of the correlations presented in Figure 4.

We observe more fast than linear and various growth of correlations at increasing rank $r$ in Figure 4(a). Rich diversity of various hinges in Figures 4(b)-(d) also excloude posibility for an unambiguous and simple identification any networks of entropy as networks of "small worlds". Therefore, hypothesis about "small worlds" structure for information entropy of DNA, as a common universal principe, here is no good. Complex hinges in Figures 4(b)-(d) and their dependence on the state of health may to serve one of illustration of various fragmentations in fractal networks. Strong variations of hinges in Figures 4(b)-(d) at variations states of health show that topology or fractal structure of entropy network has strong dependence on the states of health.

Moreover, more dense and more real types of entropy networks presented in Figure 5. Packing of data in Figures 5(a) and (b) corresponds to exponentially dense packing of "small worlds". According to Figure 5 we never have ideal networks of "exponentially small worlds" in real life, but we have a very perfect networks of "exponentially small worlds" without fractals $(D=2)$ as a clear simple standard for comparisons of deviations of various fractal correlations with this ideal standard. In Figures 5(a) and (b) are observed more ordered situa-

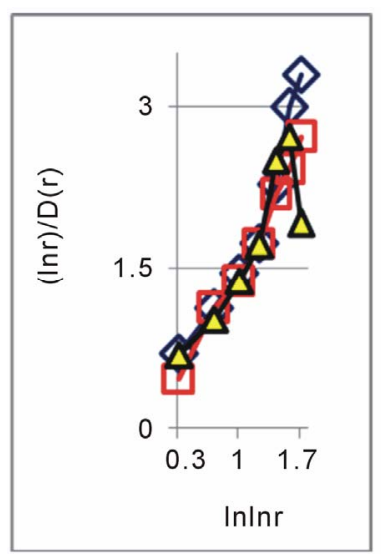

(a)

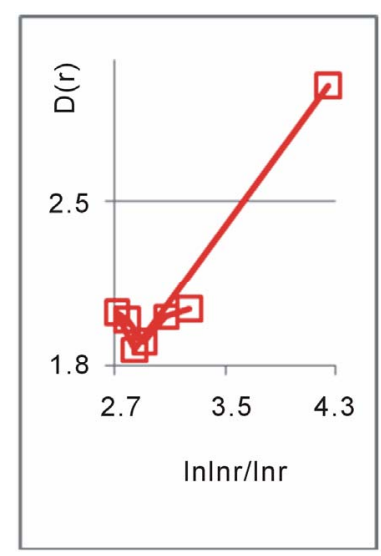

(c)

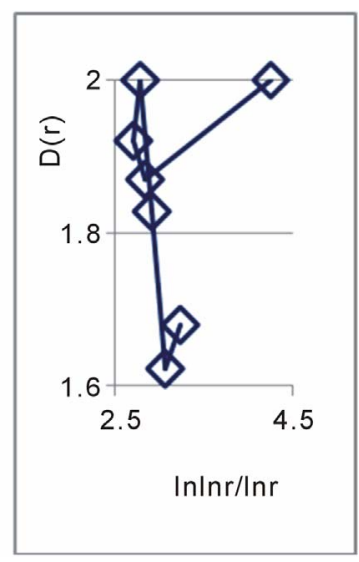

(b)

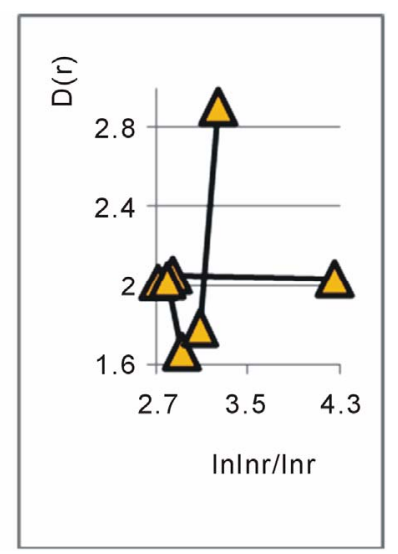

(d)
Figure 4. Large-scale distributions of fractal dimension $D(r)$ for variables corresponded to networks of 'small worlds'; (a) $(\ln r) / D(r) \sim\{\ln \ln r\}$; various hinges of fractal dimension $\mathrm{D}(\mathrm{r})$ in the dependence of $D(N) \sim \ln N / \ln \ln N$ for (b) asthma, (c) good health (d) oncology; initial histograms shown in Figure 2(b).

tions in topology structure of networks than in Figures 4(a)-(d) for networks of "small worlds". Therefore networks of "exponentially small worlds" are more suitable for description of information entropy of DNA.

Other type of correlations may be presented in networks of "double logarithm scale" in Figure 6.

According to Figures 5 and 6, branching and rather notable differences in networks corresponding to various states of health are observed for small rank $r=4$ and big rank $r>32$. Rather good coincidence and local universality of entropy networks for different states of health are observed at rank $r=8,16,32$ in Figure 5 for "exponentially small worlds" and in Figure 6 for "double logarithm scale". Stratification and individual deviations from common correlations at other values of rank $r$ depend on the states of health; variations of health status correspond to variations in informational networks of DNA activity. In Figures 5(b) and 6(b) we observe two 


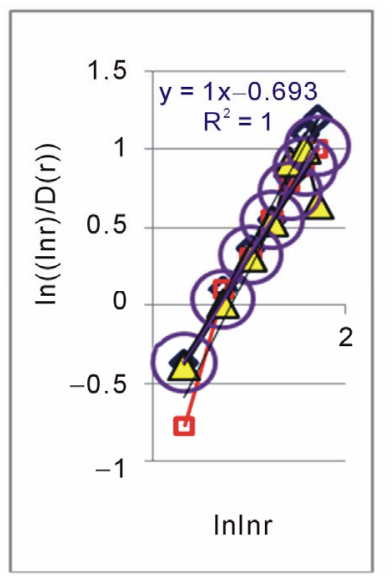

(a)

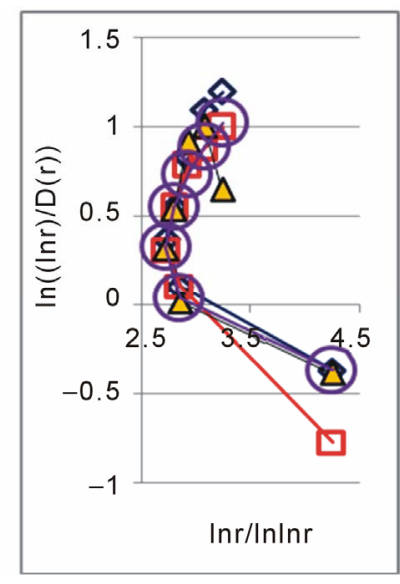

(b)
Figure 5. Dependences of fractal dimension $D(r)$ on double logarithm of range $r$ in the variables for 'exponentially small worlds' (a) $(\ln (\ln r / D(r))) \sim \ln \ln r \quad$ (b)

$\ln (\ln r / D(r)) \sim \ln r / \ln \ln r$ in multi-scale networks of "real worlds" and in networks of "exponentially small worlds" for information entropy of fluorescing DNA inside neutronphils; the ideal network of "exponentially small worlds", without fractals $(D=2)$, corresponds to violet line with round dots; initial histograms shown in Figure 2(b).

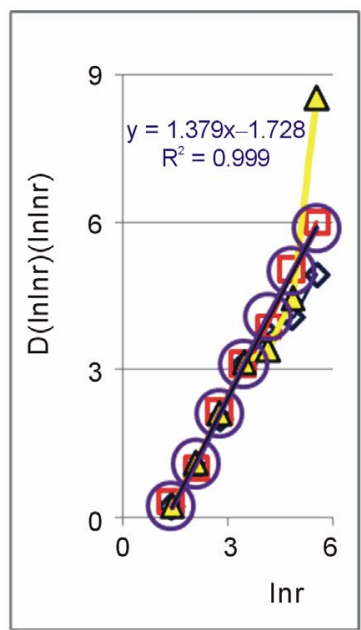

(a)

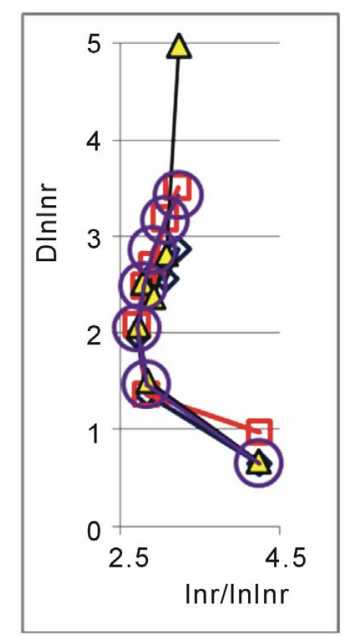

(b)
Figure 6. Dependences of fractal dimension $D(r)$ on double logarithm of range $\boldsymbol{r}$ in the variables for "double logarithmic scale" (a) $D(\ln \ln r)^{2} \sim \ln r \quad$ (b) $D \ln \ln r \sim \ln r / \ln \ln r \quad$ in multiscale networks of "real worlds" and in networks of “double logarithmic scale” for for information entropy of fluorescing DNA inside neutrophils; the ideal network of “double logarithmic scale”, without fractals $(D=2)$, corresponds to violet line with round dots; initial histograms shown in Figure 2(b).

different branches for small and big values of rank $r$ in the point of $r=16$. Stratification and branching of fractal structures for DNA information entropy shape and define various alternative ways of transmitting information. Different alternative means of information communication for the global network of DNA inside cells ensure by local networks of different clusters with the same topology (identical $D$ ) at different scales of $r$ or at varied values of entropy $(E=\ln r)$ in the dependence the states of health, as in Figure 3. The same fractal dimension $D$ can match the clusters of different scales $r$ with a different number of flashes. Currently we don't now other clear details of fragmentation. We have no universality in informational networks of DNA, only perfect etalon, as theoretical measure for estimations of information communications in the not existing in real life and ideal cells, which presented here as violet lines in Figures 5 and $\mathbf{6}$ for an ideal case $D=2$. Reality connected with variations of homeostasis regulation, i.e. with changing noises of information entropy for DNA activity during life of cell.

\section{Noise of Entropy in Homeostasis Support. Patterns and Switches for Holder's Averages}

We have no of ideal, absolute, correct homeostasis, nowhere and never. We always observe various fluctuations during regulation of stability near homeostasis. These fluctuations also very individual and consist many information on stability regulation the dynamic equilibrium in homeostasis. Let us consider various deviations, fluctuations or noise of entropy $e_{l}(r)$ near homeostasis of total information entropy $E(J, r)$

$$
e_{l}(r)=E_{l}(r) / E(J, r)-1, E_{l}(r)=-p_{l}(r) \ln p_{l}(r)
$$

Mean characteristics for individual distributions of noises in a blood sample defined by the central moments and Holder's averages for noises of entropy $e_{l}(r)$.

The central moments of $\left\langle e(r)^{m}\right\rangle$ for fluctuations of entropy $e_{l}(r)$ near homeostasis defined by the statistical averages

$$
\left\langle e(r)^{m}\right\rangle=M(e(r), m)
$$

where $m$ determines the order of moment $M(e(r), m)$. Here symbol $\langle\ldots\rangle$ denotes statistical average for fluctuations of entropy $e_{l}(r)$. The power means or averages of Hölder for deviations of entropy $e_{l}(r)$ are

$$
\langle e(m, r)\rangle=\left\{\frac{1}{r} \cdot \sum_{l=1}^{l=r}\left(e_{l}^{m}\right)\right\}^{\frac{1}{m}}
$$

Three illustrations for distribution of central moments $M(e(r=4), m)$ and of averages of Hölder $\langle e(r=4), m\rangle$ at rank $r=4$ presented in Figure 7. These distributions are determined on the base of histograms of rank $r=4$ for information entropy in Figure 8.

Two branches with even and odd numbers $m$ of central 


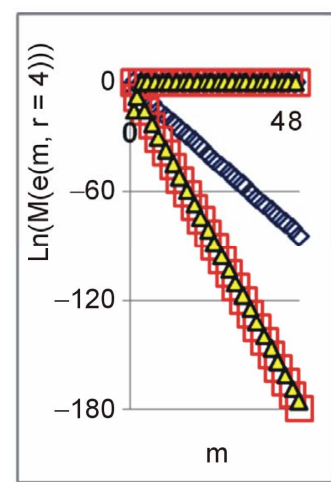

(a)

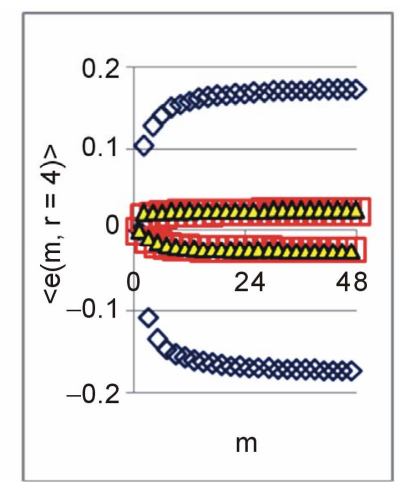

(b)
Figure 7. (a) Logarithmic distributions of central statistical moments $M(e(r), m)$ for fluctuations of entropy $e(r)$ near homeostasis for bronchial asthma (rhombuses), for a good health (quadrates), for oncology (triangles); initial histograms presented in Figure 8; lower and upper branches correspond to the even $m=2,4,6, \cdots$ and odd $m=1,3,5, \cdots$;

(b) Distributions for averages of Hölder $\langle e(m, r)\rangle$ with

different number $m$ at fluctuations of entropy $e(r)$ near homeostasis for bronchial asthma (rhombuses), for a good health (quadrates), for oncology (triangles); initial histograms presented in Figure 8; upper and lower branches correspond to the even $m=2,4,6, \cdots \quad$ and odd $m=1,3,5, \cdots$.

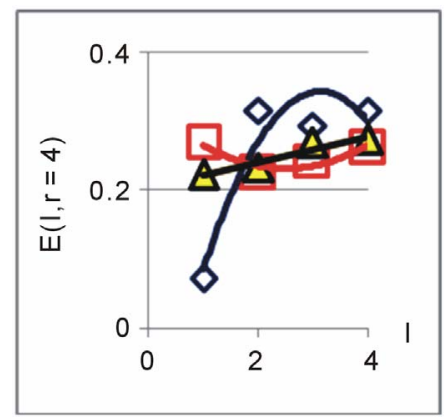

Figure 8. Dependence of normalized distributions of Shannon entropy $E(I, r=4)$ on intensity $I$ at rank $r=4$. The continuous lines correspond to the parabolic approximations of probability density distributions, three types of lines correspond to different types of stability for transcritical bifurcation in homeostasis regulation, at change the states of health; initial histograms shown in Figure 2(b).

moments $M(e(r), m)$ have unique universality with zero and clear exponential decreasing of $M(e(r), m)$, as in Figure 7(a). The exponential decreasing of $M(e(r), m)$ provides the informational stability of DNA activity. Decreasing rate of $M(m, r)$ have trends to growth at increasing numbers $m$ and $r$. A very slow growth for averages of Hölder $\langle e(m, r)\rangle$ in Figure 7(b) clearly defines individual level of fluctuations and autocorrelations of entropy $e(m, r)$ at homeostasis for given person, unlike of unified and quick degradation of central moments $M(e(r), m)$, as in Figure 7(a).

Averages of Hölder $\langle e(m, r)\rangle$ define various orders of autocorrelation, at various numbers of $m$, for fluctuations of entropy near the "centre of gravity" in stability regulation the dynamic equilibrium in homeostasis. According to Figure 7(b) all deviations of entropy, for various individual distributions of $\langle e(m, r)\rangle$ from zero level, increase with increasing the number of $m$, with various saturations of chromosomal correlations of entropy at different states of health until the value of $m=$ 46, which defines the number of chromosomes inside cells. Therefore, the value of $\langle e(m=46, r)\rangle$ is the largest among all mean. Therefore, overall level of entropy noise defined by the values of $\langle e(m=46, r)\rangle$, according to a well known inequality

$(\langle e(m-1, r)\rangle)<(\langle e(m, r)\rangle)$ for Holder's averages. This means, also, that all 46 chromosomes involved in support regulation of homeostasis inside cells, as one united full set.

All distributions of $\langle e(m, r)\rangle$ in Figure 7(b) have an oscillatory behavior at increasing of $m$. Two branches with even and odd numbers $m$ correspond to the negative and positive values of $\langle e(m, r)\rangle$. These oscillations with period 1 not specified in Figure 7(b); the mandatory behavior doesn't needing in comments.

Average noise level of information entropy in DNA activity at given range $r$ is defined by the standard deviation $\sigma(e(m, r))=\left(\frac{1}{m} \sum_{k=1}^{k=m}\langle e(k, r)\rangle^{2}\right)^{1 / 2}$ of $\langle e(m, r)\rangle$ for $m=46$. These are very important characteristics for information transfer of DNA activity inside cells. These levels of $\sigma(e(m=46, r))$ defined by all values of $m=$ 46 for all averages of Hölder $\langle e(m, r)\rangle$, but not only the lower numbers of $m=1,2$; we have 46 chromosomes in the cells and many types of cross-correlations. Noise of entropy or average noise level for one chromosome, defined by the values of $\sigma(e(m=46, r))$, in DNA activity ensures contrast in information transfer and correlations inside cells at given scale $r$ for any networks of DNA activity. Value of $\sigma(e(m, r))$ depends on health status. In Figure 7(b) maximal noise level $\sigma(e(m=46, r=4)) \approx 18 \%$ corresponds to strong inflammations at asthma.

Distributions of $\langle e(m, r)\rangle$ in Figure 7(b) depend on the health status. These distributions define stable and clear classification of noise level in networks of entropy for DNA activity at regulation of homeostasis, for given person at given time.

We observe very strong switching of branches for $r=$ 4 in the averages of Hölder in Figure 7(b) for asthma with respect the same branches for a good health and oncology. The same switching observed for distribution of central moments $M(e(r), m)$ in Figure 7(a). Analysis show that distributions of various other averages of 
Hölder, for other values of rank $r$ are rather close to each other. This means the similar noise levels of information entropy near homeostasis at oncology and in a good health for given donors at other values of rank $r$. In the latter case we observe very notable differences in fractal structures of networks of information entropy in Figures 3-6 and difference in their stability (see bellow Figure 8). Therefore, we cannot assume that similarity in the averages of Hölder linked with the coinciding characteristics of collective correlations inside and between chromosomes in networks of a certain scale $r$ for different states of health. This means only some similarity in the levels of noises for ensuring information transfer and chromosomal correlations near homeostasis, as for one and the same noise level in various Brownian motions.

Mean level of experimental errors in original cytometric histograms for $r=256$ is about 2\% [6-8]. We observe much more noticeable and very clear difference between initial and transformed experimental distributions in Figures 1 and 2 for different states of health and much more essential difference for averages of Hölder $\langle e(m, r)\rangle$ for deviations of entropy in Figure 7(b). This means that in all cells exists and maintained a very effective stabilization of homeostasis in various networks of entropy for various states of health. The origin and mechanism of this universal stabilization now are unknown. Various regulation of homeostasis must be very quick and must have the general physical origins, as for the dipole and multiple resonances for excitations in large clusters.

Switching between networks of entropy in Figures 46, corresponds to different states of health, with different deviations of $e_{l}(r)$ from homeostasis of entropy. Hidden switching between branches for averages of Hölder $\langle e(m, r=4)\rangle$ and central moments $M(m, r=4)$ in Figure 7 for a good health, oncology and for asthma here linked with changing stability due to transcritical bifurcation in distributions of information entropy in Figure 8 for different statuses of health.

\section{Statistical Stability and Transcritical Bifurcation}

Let us consider statistical stability varied distributions of information entropy following to some approaches presented in [3]. Reduction of rank $r$ leads to distribution of entropy presented in Figure 8. Large-scale distributions of information entropy for rank $r=4$ in Figure 8 have different statistical properties and different stability types for various states of health. These properties are connected with transcritical bifurcation in homeostasis regulation. Transcritical bifurcation has a normal form [15]

$$
\partial_{t} v=A v-v^{2}
$$

where $A$ is a control parameter. The dynamical system (13) has stationary solutions that correspond to fixed points. The fixed point $v_{0}=A$ depends on the sign and value of a control parameter $A$. If $A>0$ there is an attractive fixed point and stable solutions to Equation (13). If $A<0$ there is a repelled fixed point and unstable solutions to Equation (13). If $A=0$ there is a shunt fixed point and neutral stability. The introduction of the source of additive white noise reduces Equation (13) to the Langevin equation

$$
\partial_{t} v=A v-v^{2}+f(t),\left\langle f(t) f\left(t^{\prime}\right)\right\rangle=2 D^{\prime} \delta\left(t-t^{\prime}\right)
$$

where $f(t)$ is the Langevin source, $\delta\left(t-t^{\prime}\right)$ is the Dirac $\delta$-function, $D^{\prime}$ is diffusion coefficient for white noise. Linearization near the fixed point $v_{0}=A$ reduces Equation (14) to the linear Langevin equation for small deviations of $x=v-v_{0}$,

$$
\partial_{t} x=-A x+f(t)
$$

The Fokker-Planck equation that corresponds to Equation (15) is

$$
\left(\partial_{t}+A \partial_{x}+D^{\prime} \partial_{x x}^{2}\right) \rho(x, t)=0
$$

Equation (16) determines the stationary probability density $\rho_{s}(x)$ as $\rho_{s}(x) \sim \exp \left(-A x^{2} / 2 D^{\prime}\right)$. The probability density distribution $\rho_{s}(x)$ corresponds to attractor if $A>0$. The statistical determination of the attractor is used as the more probable state, when the distribution function has a maximum as convex parabola in Figure 8.

A similar determination of attractor is actually equivalent to the approach proposed in [16]. If $A<0$ there is a repelled fixed point and minimum of $\rho_{s}(x)$. Let us introduce $x=I(4)-I_{\text {extr }}(4)$, where $I_{\text {extr }}(4)$ corresponds to the position of minimum or maximum for curves in Figure 8. The attractor fixed point corresponds the upper parabola in Figure 8 if $\left(A x^{2} / 2 D^{\prime}\right) \rightarrow 0$. This parabola characterizes the blood immunofluorescence at inflammatory diseases; in our case this is bronchial asthma.

The lower parabola in Figure 8 corresponds to unstable $(A<0)$ critical point, characterizes a good health. Fixed point of neutral stability with the ideal value of $A=0$ was not observed. Instead, a large family of lines with very small positive and negative curvatures for various autoimmune and oncology diseases without inflammations is observed, as the line passing through triangular points in Figure 8.

If one considers the curvature or curvature radius of parabolic approximations of entropy $E(I, r=4)$ in Figure 8 as the bifurcation parameter $A$, then various types of statistical distributions of $E(I, r=4)$ can be classified in the frame of transcritical bifurcation. Proposed criteria correspond to informational homeostasis of entropy. The bifurcation corresponds to stability change in homeostasis regulation for various health statuses. Thus, three types of informational homeostasis correspond to 
positive, negative and neutral stability for distributions of information entropy at oxidative activity of DNA inside cell. The bifurcation reflects the collective statistics effects of various cellular processes and switching of transitions from health to illness and from illness to health.

\section{Changeability and Switching of Entropy's Noise for a Healthy Donor in Real Time}

Let us compare, in real time, during one year, Shannon entropy for DNA activity inside cells of healthy donor.

Three histograms are shown in Figure 9.

One may to compare histograms in Figures 1 and 9. Distributions of entropy are shown in Figure 10.

One may to compare histograms in Figures 2 and $\mathbf{1 0 .}$

Fractal peculiarities of entropy are shown in Figure 11.

Dense packing of entropy is shown in Figure 12.

Some features of stability and noises of information entropy at low rank $r=4$ are shown in Figure 13.

One may to compare histograms in Figures $\mathbf{1}$ and $\mathbf{9 ,} 2$ and 10, 4 and 11, 6 and 12, 7 and 13(b), 8 and 13(a). We observe changes in distributions of entropy and fractal structures, noise level etc. in real time for one and the same healthy man and in the comparisons with various distributions for different unhealthy people. Many of fractal peculiarities of entropy change in time and depend on health status. Constancy belongs to homeostasis or to invariance of total Shannon entropy in Figures 2(c) and 10(b) for any states of health of any human. Constancy also belongs to statistical instability (positive curvature of all approximations) for entropy distributions of unchangeable healthy man in Figures 8 and 13(b) during one year. This is example of a very good immunity, as and perfect closeness to the ideal of networks in Figure 12.

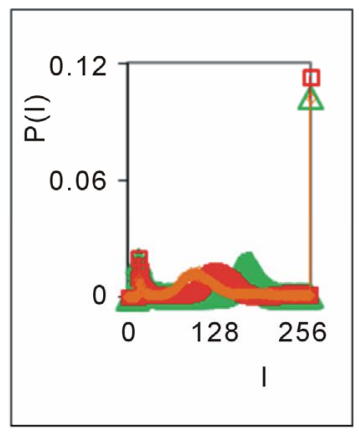

(a)

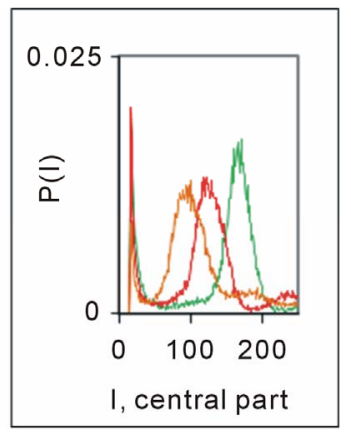

(b)
Figure 9. Dependence of normalized frequency distribution of flashes $P(I)$ on their intensity $I$ (a) and for clearest only central part of histogram (b), for one and the same invariably healthy, donor in different times. Triangle green points correspond to the total flashes number $N_{0}=30$ 832, analysis time is 19 July (first year); rhombus yellow points correspond to the total flashes number $N_{0}=38758$, analysis time is 11 July (next year); square red points correspond to the total flashes number $N_{0}=40109$, analysis time is 03 June, before 11 July, histogram range $r=256$, as in Figure 1 .

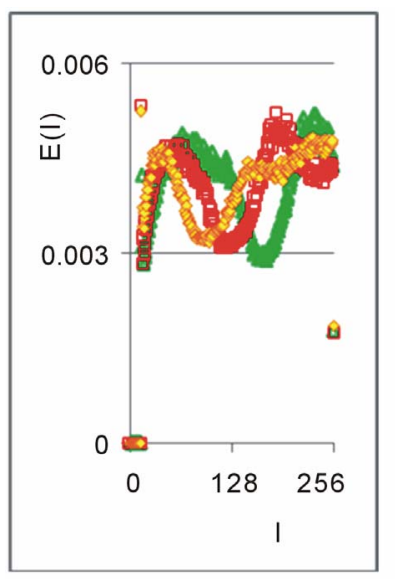

(a)

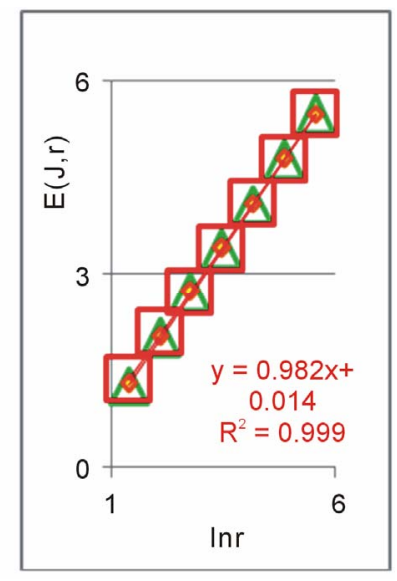

(b)
Figure 10. (a) Normalized distributions of information entropy $E(J(I))$ in the dependence on fluorescence intensity at rank $r=256$; (b) Dependence of total Shannon information entropy $E(J, r)=\ln r$ on logarithm of range $r$; initial histograms at range $r=256$; initial histogram shown in Figure 9.

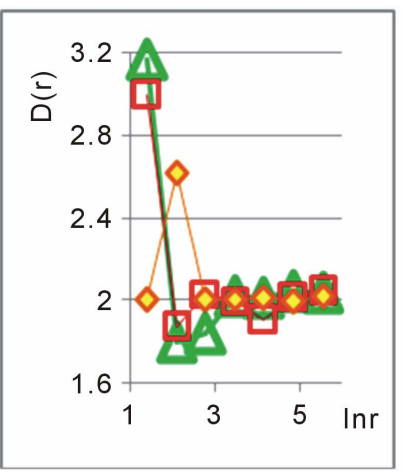

(a)

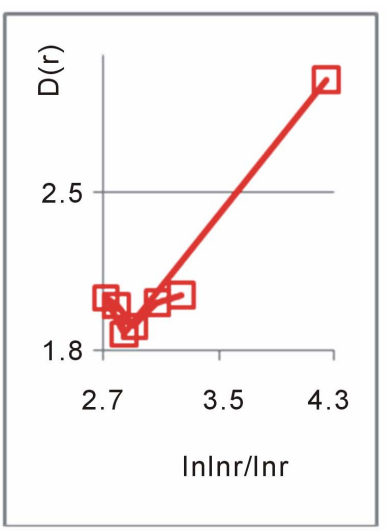

(c)

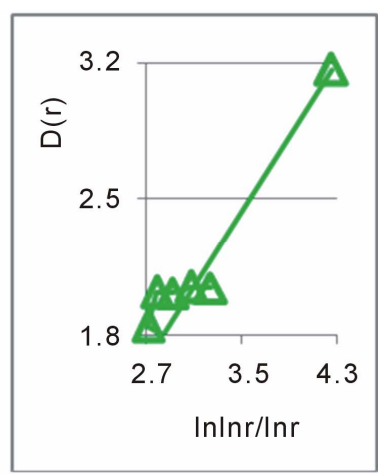

(b)

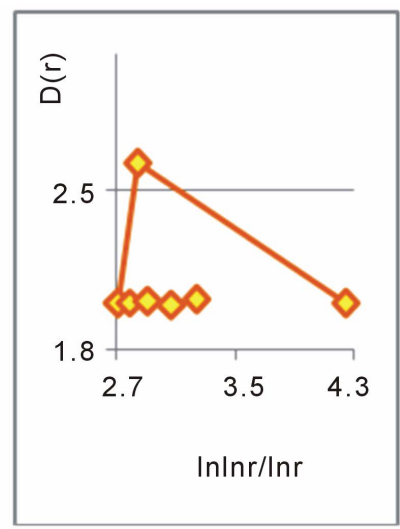

(d)
Figure 11. Distributions of fractal dimension $D(r)$ in the dependence on rank $r$ ((a)-(d)); ((b)-(d)) various hinges of fractal dimension $D(r)$ in "small world" network, in the dependence of $D(N) \sim \ln N / \ln \ln N$, for a healthy man in real time, during one year; initial histograms shown in Figure 9. 


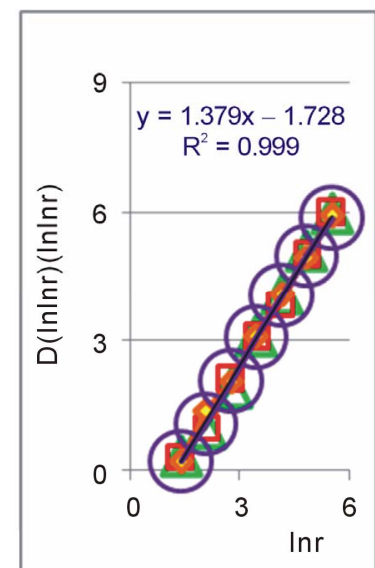

(a)

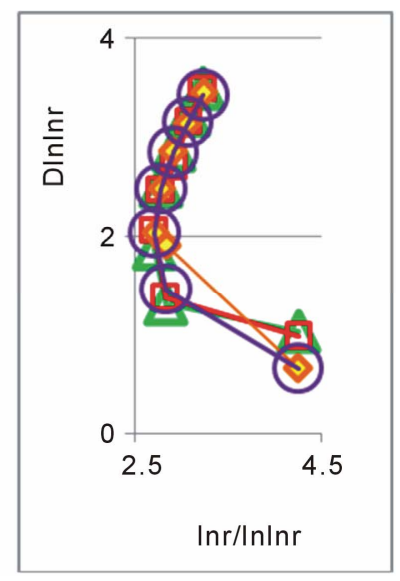

(b)
Figure 12. Dependences of fractal dimension $D(r)$ on double logarithm of range $\boldsymbol{r}$ in the variables for "double logarithmmic scale” (a) $D(\ln \ln r)^{2} \sim \ln r$ (b) $D \ln \ln r \sim \ln r / \ln \ln r$ in multi-scale networks of "real worlds" and in networks of "double logarithmic scale" for information entropy of fluorescing DNA inside neutrophils; the ideal network of "double logarithmic scale", without fractals $(D=2)$, corresponds to violet line with round dots; initial histograms shown in Figures 9 and 11(a).

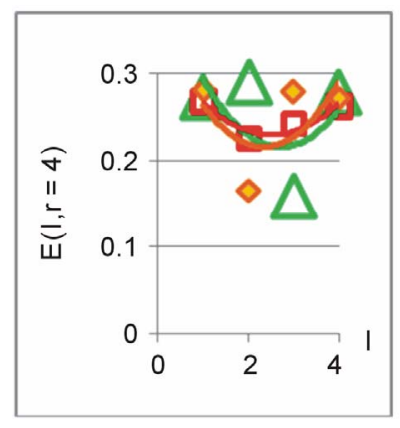

(a)

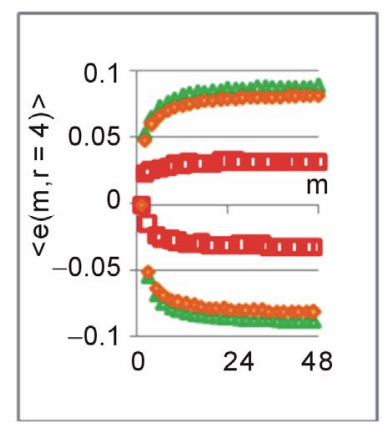

(b)
Figure 13. (a) Dependence of normalized distributions of Shannon entropy $E(I, r=4)$ on intensity $I$ at rank $r=4$. The continuous lines correspond to the parabolic approximations; initial histograms shown in Figures 9 and 11(a); (b) Distributions for averages of Hölder $\langle e(m, r)\rangle$ with different number $m$ at fluctuations of entropy $e(r)$ near homeostasis for a good health man in real time, during one year; initial histograms shown in Figure 9; upper and lower branches correspond to the even $m=2,4,6, \cdots$ and odd $m=1,3,5, \cdots$.

\section{Conclusions}

We study various large-scale distributions of Shannon entropy, based on normalized distribution of information in original histogram for frequency of flashes in different blood samples. We observe only one unified value of total Shannon entropy $E(J, r)=\ln r$, for all rank $r$, like the empirical invariant, as the identical sum in each given distribution of information entropy, in all samples of blood and any donor, as in Figures 2(c) and 10(b) in real time. This invariant defines the informational homeostasis of oxidative activity of 3D DNA for full set of chromosomes inside living neutrophils, in all clusters of all multi-scale networks for information activity of DNA inside cells. This invariant gives Shannon entropy as general characteristics for definition of information activity of 3D DNA in cells for any person, at any state of health, for any genome as the overall zero level for analysis and comparison chromosomal activity and DNA networks in cells for different people (Figures 5-8) and for one and the same human in different times (Figures 12 and 13).

Invariance of total entropy $E(J, r)=\ln r$ defines special role of distribution of Shannon entropy, as it is for all functions associated with the conservation laws (pulse, energy, charge etc.), as the main dominant variable to describe the states and dynamics of informational activity of DNA in cells. Nevertheless, informational invariant of $E(J, r)=\ln r$ is difficult to compare with the conservation laws of mass, energy, etc.; we have no different values of total entropy in different cells. Invariance of entropy reflects the unchangeable measure or quantitative units of information entropy at certain scale $r$ of correlations at DNA activity inside all and any cells.

Informational homeostasis reflects a number of fundamental phenomena in information and physics of real life of 3D DNA inside cells. Shannon entropy of 3D DNA in cells has much more dense packing of correlations than in well known networks of 'small worlds, than in all and any technical and computer systems. As the rule, networks of entropy are characterized by various mix of normal $D<2$ and abnormal $D>2$ fractal dimensions (Figures 3 and 11(a)) and new types of fractal patterns and fractal hinges for various fractal topology at different states of health (Figures 4-6 and 12).

Results generalized in the double logarithmic scales, in the triple and quadruple logarithmic scales, etc., as a reflection of complexity, what permits, also, detect a fragmentation, as it is shown in Figures 4-6 and 12.

Deviations or noises of information entropy $e_{l}(r)$ from homeostasis level define regulation of informational homeostasis, information transfer and information flow inside cells for different states of health. The coincidences and switching patterns in branching sequences for central moments $M(e, m)$ and for averages of Hölder $\langle e(m, r)\rangle$ of these noises are shown in Figures 7 and 13(b). We observe various saturations for averages of Hölder $\langle e(m, r)\rangle$ in the levels of chromosomal correlations of entropy at different states of health in Figures 7(b) and 13(b), at increasing number of correlations m. All chromosomes are interconnected and involved in the support regulation of homeostasis; saturation of 
correlations exists if number of $\mathrm{m}$ for average of Hölder $\langle e(m, r)\rangle$ equals to $m=46$, when all 46 chromosomes involved in homeostasis regulation. The levels of saturations are very important characteristics of inner and inter chromosomal correlations, as asymptotic values the standard deviation of $\sigma(e(m=46, r))$, which characterizes average noise level of entropy for one chromosome, for averages of Hölder. This level of saturation characterizes background noise and information transfer of DNA activity inside cells in support regulation of homeostasis, comparisons and detections various abnormalities for noises and fluctuations of information entropy of DNA in various blood sample in diagnostics. For instance, noise level of entropy near homeostasis may be rather high and to reach $\sim 20 \%$ for strong inflammations, as it observed for bronchial asthma in Figure 7(b).

We defined manifold of chromosomal networks of entropy in Figures 4-6 and 12 and (or) many ways for homeostasis regulation. We show that regulation of homeostasis belongs to one from three classes of stability for all statistical distributions of information entropy; negative, neutral and positive stability for healthy people and dominants of autoimmune or inflammation diseases. Changes of stability, associated with transcritical bifurcation, are shown in Figure 8.

To be continued.

\section{Acknowledgements}

Thanks to M. Filatov for kindly providing the experimental data.

\section{REFERENCES}

[1] M. V. Filatov, E. Y. Varfolomeeva and E. A. Ivanov, "Flow Cytofluorometric Detection of Inflammatory Processes by Measuring Respiratory Burst Reaction of Peripheral Blood Neutrophils," Biochemistry and Molecular Medicine, Vol. 55, No. 2, 1995, pp. 116-121. http://dx.doi.org/10.1006/bmme.1995.1041

[2] N. E. Galich and M. V. Filatov, "Laser Fluorescence Fluctuation Excesses in Molecular Immunology Experiments," Proceedings of the Society of Photo-Optical Instrumentation, Vol. 6597, 2007, Article ID: 65970L.

[3] N. E. Galich, "Bifurcations of Averaged Immunofluorescence Distributions Due to Oxidative Activity of DNA in Diagnostics," Biophysical Reviews and Letters, Vol. 5, No. 4, 2010, pp. 227-240. http://dx.doi.org/10.1142/S1793048010001196
[4] N. E. Galich, "Cytometric Distributions and Wavelet Spectra of Immunofluorescence Noise in Medical Diagnostics," World Congress on Medical Physics and Biomedical Engineering, Munich, 7-12 September 2009, pp. 1936-1939,

[5] N. E. Galich, "Shannon-Weaver Biodiversity of Neutrophils in Fractal Networks of Immunofluorescence for Medical Diagnostics," Journal of WASET, Vol. 70, 2010, pp. 504-515.

http://www.waset.org/journals/waset/v45/v45-92.pdf

[6] N. E. Galich, "Complex Networks, Fractals and Topology Trends for Oxidative Activity of DNA in Cells for Populations of Fluorescing Neutrophils in Medical Diagnostics," Physics Procedia, Vol. 22, 2011, pp. 177-185. http://dx.doi.org/10.1016/j.phpro.2011.11.028

[7] N. E. Galich, "Informational Homeostasis for Shannon Entropy in Complex Networks of Oxidative Activity of DNA in Cells; Fractals, Stability and the Switching in Large-Scale Gene Nets for Fluorescing Neutrophils in Medical Diagnostics," World Congress on Medical Physics and Biomedical Engineering, Beijing, 26-31 May 2012, pp. 542-545.

[8] J. Feder, "Fractals," Plenum Press, New York, 1988. http://dx.doi.org/10.1007/978-1-4899-2124-6

[9] T. Gneiting and M. Schlather, "Stochastic Models That Separate Fractal Dimension and the Hurst Effect," SIAM Review, Vol. 46, No. 2, 2004, pp. 269-282. http://dx.doi.org/10.1137/S0036144501394387

[10] B. Mandelbrot, "The Fractal Geometry of Nature," W.H. Freeman, San Francisco, 1977.

[11] D. J. Watts and S. H. Strogatz, "Collective Dynamics of Small-World Networks," Nature, Vol. 393, No. 6684, 1998, pp. 440-442. http://dx.doi.org/10.1038/30918

[12] L. A. N. Amaral, A. Scala, M. Barthélémy and H. E. Stanley, "Classes of Small-World Networks," Proceedings of the National Academy of Sciences, Vol. 97, No. 21, 2000, pp. 11149-11152. http://dx.doi.org/10.1073/pnas.200327197

[13] A. Wagner and D. A. Fell, "The Small World inside Large Metabolic Networks," Proceedings of the Royal Society of London. Series B, Vol. 268, No. 1478, 2001, pp. 1803-1810. http://dx.doi.org/10.1098/rspb.2001.1711

[14] M. E. J. Newman, "The Structure and Function of Complex Networks," SIAM Review, Vol. 45, No. 2, 2003, pp. 167-256. http://dx.doi.org/10.1137/S003614450342480

[15] Y. A. Kuznetsov, "Elements of Applied Bifurcation Theory," Springer, New York, 1995.

[16] N. G. Van Kampen, "Stochastic Processes in Physics and Chemistry," North-Holland Personal Library, 1984. 